\title{
Fall Detection Algorithm for the Elderly Based on Trial Acceleration and Heart Rate
}

\author{
Meng Weibin, Wang Chenchao, Li Haoyang, Ji Meichen \\ Jilin University, Changchun Jilin 130012 \\ 1234@163.com
}

Keywords: the elderly, gesture detection, heart rate.

Abstract: The paper introduces a method of judging the gesture according to triaxial acceleration data and heart rate data. The acceleration transducer is used to collect the triaxial acceleration data, and some experimental data is selected as training data. The collected data is classified according to KNN algorithm, and benchmark data is designed in each classification. The feature of falling data is achieved by comparing gesture data. For application, the matching algorithm should be designed, and the similarity degree with benchmark data should be computed. Lastly, the heart rate is used for judgment and is weighted to get the matching results.

\section{Introduction}

The 21st century is called Silver Century. The sixth population census data in 2010 indicates that there were more than 120 millions of old men who were greater than 65 years old, which accounts for $8.87 \%$ of the total population, and rises by $1.91 \%$ compared with that in 2000 . According to the prediction, there are 350-450 millions of old men in 2040 in China [1]. Under the condition of growing aging, the life health and safety problem of the elderly is more and more evident. The investigated data indicates that the main reasons for accidental death of the urban elderly include accidental falls, traffic accidents and suicide [2]. And it is untimely rescue after accidents that causes death. In order to solve the dilemma, the paper proposes the schema of monitoring the health status of the elderly in real time and timely alarming when there are accident. At present, the monitoring technique for the elderly in China is in the stage of passive care and rescue after accidents [3]. Intelligent health watch for the elderly of Syshelp Company has the functions of GPRS positioning, heart-rate detection and communication [4]. Hi-Watch intelligent health cloud watch of Heal Health Technique Limited Company has the functions of motion record, heart-rate detection and respiratory rate detection [5]. For monitoring gesture and body state, there are relevant researches. For example, "863” Planning Support Project in China includes modularized three-dimensional gesture measurement and body parameter sensing function components. But the above research has the problems of single function (the main function is detection) and single judgment way for the state of the detected objects. And only depending on single indicator such as acceleration or heart rate is easy to make greater error.

For the above phenomenon, the paper designs the portable device integrating acceleration sensor, heart-rate sensor and GSM/GPRS for collecting the real-time acceleration and heart rate information. And the paper designs algorithm to recognize and judge the gesture and health state of the carrier. It automatically positions and sends the signals for help when the wearer needs rescue. The paper mainly introduces how the device judges the gesture and health status of the elderly.

\section{Fall Recognition Algorithm}

Fall Recognition. Fall means sudden, heteronomous and unpremeditated postural change, or falling down to the ground. From the perspective of physiology, fall is the representation that the balanced capacity of the human body declines. The statistical data indicates that the main reasons for fall of the elderly include weak legs (38\%), stumble (23\%), slippery ground (9\%), falling in a faint (7\%) and missing step (6\%) [6]. During shorter period (the continuous time of most falls is 
1-6s [7]), the acceleration and angular velocity of human body may change greatly. The paper collects the acceleration and angular velocity of human body with different gestures. After processing and analyzing, the standard acceleration change feature diagram is achieved. The real-time collected acceleration and change mode of human body is compared with standard features, which can get the judgment for the gesture of human body.

For computation, we mainly use the feature value SMV (Signal Magnitude Vector) as characteristic quantity to reflect the acceleration of human body. Acceleration is the vector and has directions, so $S M V=\sqrt{a_{x}{ }^{2}+a_{y}{ }^{2}+a_{z}{ }^{2}}$ can ignore the influence of the directions of acceleration, which is good for judging the same gesture in different directions. $a_{x}, a_{y}$ and $a_{z}$ means the acceleration value of $\mathrm{X}, \mathrm{Y}$ and $\mathrm{Z}$.

1. Analysis of multi-motion acceleration

Before analyzing the falling state, the paper firstly analyzes the common activities in daily life. Other activities can be used to guess the acceleration change of falling. The paper analyzes the acceleration change of three motions, squat and stand up, ascend the step and continuous jump.

Firstly, the paper analyzes the motion of squat and stand up. Squat is that the body firstly is down and weightless, and is decelerated, which is overweight. So squat is weightless firstly and then is overweight. Similarly, standing up is firstly overweight and then is weightless. Three times of squat and stand up is made in 25 seconds. The acceleration change of three directions and SMV is shown in Figure 1. Blue, purple, green and red lines mean the values of SMV, $x$ axis, $y$ axis and $z$ axis. The above analysis indicates that squat and standing up is the process of weightless, overweight, overweight and weightless. In the figure, it is manifested as trough, crest, crest and trough, which indicates that SMV reduces when it is weightless, and SMV increases when it is overweight.

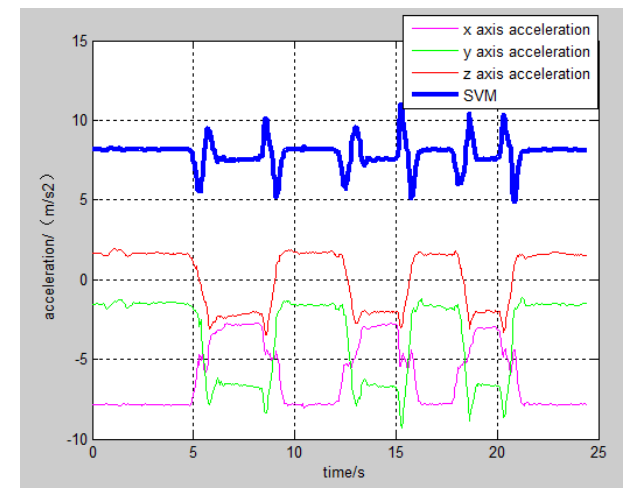

Figure 1 Continuous squat \& stand up

Then, the paper analyzes the action of ascending the steps. Figure 2 is the curve of the change of ascending two steps. The hind leg bends for the first step, and the gravity center falls, which is overweight. And SMV reduces. Then, the forward foot takes a step, the hind leg energizes, and the ground pressure increases, which is overweight. And SMV achieves a peak. After taking a step, the gravity center translates, and SAV shocks, which transits to the next step. And the above change is repeated.

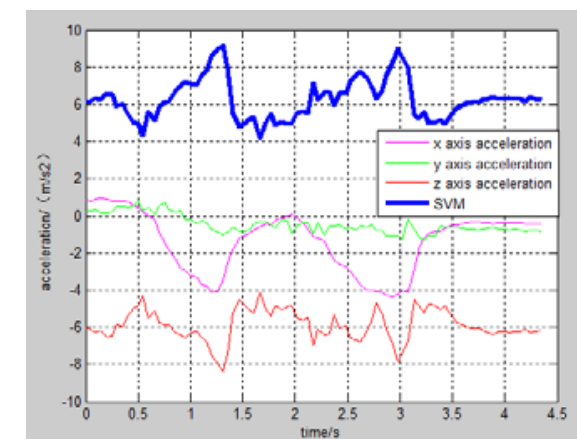

Figure 2 Continuous ascend the steps 
In the action of continuous jump, firstly, the gravity center falls down and SMV reduces. Then, the foot takes a run jump, which makes the ground pressure increase and SMV increase. The next stage is falling down to the ground from the peak. The body is overweight before falling down to the ground, and SMV reduces continuously and achieves the minimum until the foot falls down to the ground. Lastly, the ground pressure and SMV increases, which is an overweight process.

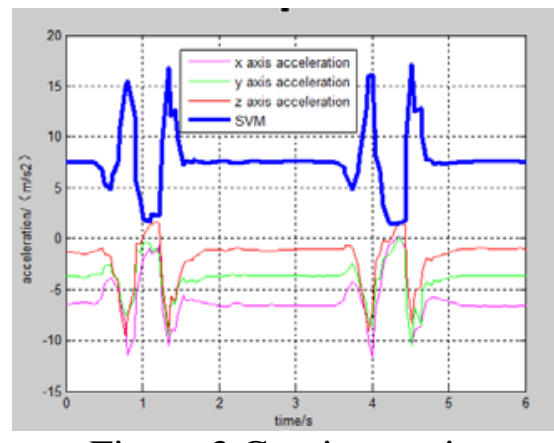

Figure 3 Continuous jump

2 Acquisition of reference acceleration curve of fall

From the above analysis on three actions, we can infer that there is similar SMV change forfall. The paper divides the falling process into three parts.

Overweight stage. In the stage, the human body is overweight because of losing balance. In the state, the figure line of SMV sinks and achieves a trough.

Impact stage. In the stage, the human body and ground collides with other planes. The reaction of the planes makes the curve of SMV fluctuate greatly which is greater than the daily activity value, and makes it achieve the peak. And three axis are in the great fluctuation state with SMV.

Peace stage. In the stage, after falling and colliding with the plane, the elderly can't stand up at once, which is a smooth state. It indicates that fall is over. The change of SMV curve for a falling sample is shown in Figure 4.

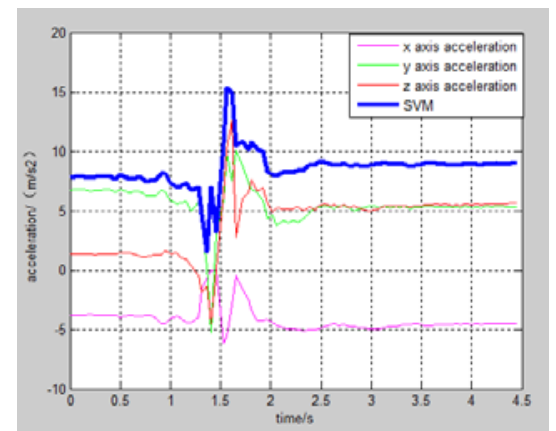

Figure 4 Fall

The paper compares the acceleration and the change of SMV of four motions, as shown in Table 1. The information in the table is similar to the data, and the continuous time of most falls is 1-6s [3]. The maximum and the minimum of continuous jump is similar to that of SMV, but fall has peace stage, and SMV is smooth for a long time, which makes it easy to differentiate the curve of continuous jump and it. From the statistic information in the table, we can see that it is easy to differentiate the other actions.

\begin{tabular}{|l|l|l|l|l|}
\hline Experiment Type & $\begin{array}{l}\text { Duratio } \\
\mathrm{n}\end{array}$ & SMV Max & SMV Min & Single shape \\
\hline Fall & $>1.5$ & 15.3 & 2 & Low->High \\
\hline Squat \& stand up & $>5$ & 11.1 & 4.9 & $\begin{array}{l}\text { Low->High->High- } \\
>\text { Low }\end{array}$ \\
\hline Ascend the steps & $>1.5$ & 9 & 4.1 & Low->High \\
\hline Jump & $>1$ & 17.5 & 1.9 & $\begin{array}{l}\text { Low- }>\text { High->High- } \\
>\text { Low }\end{array}$ \\
\hline
\end{tabular}


The paper makes many falling tests with different angles, directions and continuous time. The collected data is used as training data. KNN (K Nearest Neighbor) [4] algorithm is used to classify falls. For detection, the collected data is classified, and is compared with the standard curve to get the judgment.

3 Comparison of real-time acceleration and benchmark acceleration

Firstly, the acceleration sensor is used to achieve an acceleration which achieves the threshold and has the weightless feature as the sign of beginning to match. And the peace acceleration achieving the threshold in the presetting time is the sign of ending. The time for $5 \mathrm{~s}$ before beginning and ending is marked as $\mathrm{T}$, and the acceleration value in the interval is collected to generate a binary set including time and instantaneous acceleration. The time domain Ts of benchmark acceleration curve is zoomed to make it equal to Tt. The standard deviation of the acceleration value in each sampling time and that of a sampling point at the same time is computed, and the greater standard deviation corresponds to the lower matching degree.

Especially, the sampling point near the trough and peak of benchmark acceleration curve receives special process. If the sampling value is lower than the benchmark value in trough, or the sampling value is higher than the benchmark value in peak, the matching degree increases with the increase of deviation degree, and the computation method is (sampling value-benchmark value ${ }^{*}$ s-change curve * weighted value distributed by points, and it is changed into mathematical expression, the average deviation of each point is $\mathrm{P}=\sqrt{\frac{1}{n} \sum_{i=1}^{n}\left[\left(x_{i}-\mu_{i}\right)^{2}\right]}$.

The standard deviation is processed by blocked functions to make the expression of matching value of each point as follows, $\mathrm{ri}=\frac{1}{1+\left[\left(x_{i}-\mu_{i}\right)^{2}\right]^{t}}$;

The expression of the matching value of the crest and the trough point is ri $=$ $\left(\left|x_{i}-\mu_{p}\right|-\left|\mu_{i}-\mu_{p}\right|\right) * \frac{1}{1+x^{t}}$.

The matching result value of the curve is achieved. And the valuation function is $\mathrm{R}=\frac{1}{n} \sum_{i=1}^{n} r_{i}$

$\mathrm{n}$ is the number of sampling points of standard figure line, $\mathrm{xi}$ is the sampling value of acceleration of points, $\mu_{i}$ is the value of standard figure line at point I, and $\mu_{p}$ is the acceleration value in peace stage.

4 Heart-rate detection assists weighted algorithm

If the matching result of the other algorithms can't reach the threshold, the system applies heart-rate detection algorithm to assist judgment. Heart-rate detection matches sampling heart rate and irreversible shock heart rate. The matching way is consistent with the gesture detection, which is not repeated here.

The paper mainly collects the heart rate of the wearer for a long time to generate standard heart-rate status of the wearer. And the standard status is used to modify the irreversible shock heart rate curve which is achieved from the literatures, which gets the irreversible shock heart rate feature curve of the wearer. The matching value is achieved according to the computation value of each point. The matching value which is achieved by gesture recognition algorithm is weighted to get the new judgment value to determine if there is need to alarm.

The weighting algorithm is $F=R+\frac{1}{k+R^{d}{ }_{h}}$

$\mathrm{F}$ is the final judgment value, and if it is greater than 1 , it means alarm. Rt is the matching result value computed by gesture detection algorithm, and $\mathrm{k}$ is the parameter and is 1.97 . $\mathrm{Rh}$ is the matching result value computed by heart-rate detection algorithm, and $\mathrm{d}$ is the parameter and is 2.02 . 


\section{Experimental Test}

The experiments use the collected acceleration data. Heart-rate data is difficult to be achieved because of shocked feature, so the paper uses simulated data. In 200 experiments, we test eight motions for 25 times, squat and stand up, jump, run slowly, ascend the stairs, walk down the stairs, fall forward, fall back and fall in lateral direction, and the test data is shown in Table 2.

Table 2 the test data

\begin{tabular}{|l|l|l|l|l|}
\hline NO & Rt & Rh & Result & Sucess or not \\
\hline 1 & 0.85 & - & 0.85 & Yes \\
\hline 2 & 0.70 & 0.70 & 1.12 & Yes \\
\hline 3 & 0.53 & 0.64 & 0.95 & Yes \\
\hline 4 & 0.34 & 0.71 & 0.74 & Yes \\
\hline 5 & 0.60 & 0.70 & 1.00 & No \\
\hline
\end{tabular}

In 200 experiments, there are 21 mistakes, and the inspection accuracy is 89.5, which indicates that the accuracy of the algorithm in the paper is higher.

\section{Conclusions}

For the characteristics that the somatic function of the elderly declines, and they are easy for accidents, the paper proposes to use trial acceleration sensor to monitor acceleration information of the elderly in real time, and designs the algorithm to judge the gesture of the elderly. Combined with the heart-rate sensor, the accurate judgment for the state of the elderly is achieved.

In the research, we can get the following achievements. By comparing and analyzing daily activities and SMV curve feature of falls, we can get the basic criterion differentiating daily activities and falls. By collecting lots of experimental data and selecting some data as training data which receives the process of KNN algorithm, we can get the classification of different falling ways. By using Matlab to analyze experimental results, the paper designs matching degree computation algorithm to compute the matching degree of sampling data and benchmark data, and the accuracy is verified by experiments. The matching degree computation algorithm in the paper is promoted and improved, which can be applied the matching and recognition of many modes, and has wider application prospect.

\section{Reference}

[1] Analysis on accidental death for the urban elderly, Preventative Medicine, 2003, 30 (2)12-17.

[2] Data of the sixth population census in China, www. npc. gov. cn/npc/zgrdzz/2012-05/03.

[3] Garrett Brown, An Accelerometer Based Fall Detector: Developmen, Experimentation, and Analysis [J]. EECS/SUPERB, 2005

[4] IEEE TRANSACTIONS ON PAITERN ANALYSIS AND MACHINE INTELLIGENCE, VOL. 18, NO. 6, JUNE 1996

[5] Rougier, Caroline. Fall Detection from Human Shape and Motion History using Video Surveillance. 21 st International Conference on Advanced Information Networking and Applications Workshops, 2007'875 - 880.

[6] Shi Xin, Research on fall detection system based on pressure sensor [J], Journal of Instrument and Meter, 2010, 3: 24 - 28

[7] Winters, J. M. Emerging rehabilitative telehealthcare anywhere. Was the Homecare Technologies Workshop visionary. RESNA Press, 2002:6, 95-111. 
[8] Zhu Yueshu, Yuan Haobin, Chen Lei, Investigation of risk factors for falling of the elderly [J], Nursing Practice and Research, 2007, 4 (10): 5-7. 